\title{
STaRS: Space Science Teaching and Resource Site
}

\author{
Glen Mackie \\ Carter Observatory, PO Box 2909, Wellington, New Zealand \\ Present address: School of Chemical and Physical Sciences, \\ Victoria University of Wellington, PO Box 600, Wellington, New Zealand \\ Glen.Mackie@vuw.ac.nz \\ http://www.vuw.ac.nz/ mackie
}

Received 1999 August 19, accepted 2000 April 15

\begin{abstract}
A World Wide Web site has been developed by astronomers and educators at Carter Observatory. STaRS is the Space science Teaching and Resource Site (www.vuw.ac.nz/ mackie/ STaRS.html) and its objectives are to (1) present astronomy investigations for 8 to 12 year olds and (2) promote topical astronomy and space science studies for a general audience. The educational investigations drawn from local and international sources and the resources provided to teachers are described. The structure and content of the general audience pages are summarised. Details of site promotion and usage and critical comment from users and educators are given.
\end{abstract}

Keywords: astronomy education

\section{Introduction}

STaRS, the Space science Teaching and Resource Site (www.vuw.ac.nz/ mackie/StaRS.html), was a pilot programme developed by astronomers and educators at Carter Observatory and was funded by the Science and Technology Promotion Fund of the Royal Society of New Zealand. This fund supports activities that promote science and technology as offering rewarding education and career options, and enables public debate to be informed by and about science and technology.

The preferred target audiences of the fund are (in order of priority) (1) children 8 to 12 years of age and their key influencers in parents and teachers; (2) senior secondary students and their key influencers in parents, teachers and career advisors; and (3) the general public. STaRS is targeted at the first and third classes of these preferred audiences.

Carter Observatory already provides an astronomy education service (www.carterobs.ac.nz/carter_educat. html) (Leather et al. 1998) for visiting school groups. Some pre-existing classroom activities have been modified for use in STaRS, new activities have been developed and existing activities on the World Wide Web have also been selected.

STaRS was also developed to increase the visibility of New Zealand astronomy and astrophysics programs and research to all New Zealanders. In October 1998 the professional astronomy community of New Zealand presented a ten-year strategy entitled 'New Zealand Astronomy and Astrophysics in the first decade of the new millennium' (see Cottrell \& Mackie 1998 and www.vuw.ac.nz/ mackie/FP/Astron_Astrophys_text. html) to the Ministry of Research, Science and Technology Foresight Project.

One of the main strategy recommendations was 'Improved public promotion and outreach' that included closer links between the professional astronomy sector and New Zealand astronomy educators, and improved outreach of research and technology results to the public, education and industry sectors.

The web is a powerful tool for astronomy education and promotion. New Zealand is an ideal country for its use because of a number of contributing factors. It has one of the highest per capita levels of Internet connectivity (both public and schools); since 1995 astronomy has been an integral part of the national schools curriculum at all levels (Leather et al. 1998); and it has a poor level of research funding in comparison with similar population and socio-economic countries (Cottrell \& Mackie 1998). Recent related articles about the web include Pasachoff (1998) on its role in astronomical teaching and Mackie (1999) (www.vuw.ac.nz/ mackie/ astsurf/top.html) on its efficient use and resources useful to New Zealand astronomers.

STaRS version 1.0 was released in March 1999. STaRS version 2.0 was released in June 1999 (the end date of the Science and Technology Promotion Fund contract) and includes design and content changes based on user comments over the three-month life of version 1.0 .

\section{STaRS Astronomy Activities and Investigations for 8 to 12 Year Olds}

The STaRS web site comprised two main sections. The first section, Astronomy for 8 to 12 year olds (www.vuw.ac.nz/ mackie/SciTechFund/STaRS/ Educ 8_12/education.html), offers links to both astronomy activities and resources.

\subsection{General Considerations}

The presentation of astronomy activities on the web benefits from extra thought more so than in the case of standard activities used in classes. The web is of course viewable outside of class by students and non-students. It is therefore important to include activities that can be done outside of the classroom and class times. The most obvious are activities based on night-time observing. 
As well, STaRS will be accessed by students without teacher supervision, and hence all information and activities should be clearly stated and easy to perform. Whilst STaRS activities are aimed at an 8-12 year old audience, it is also important to offer activities at higher levels to challenge students (and others) and to encourage extra investigation and resource work.

\subsection{Information for Teachers}

Several important pieces of information are stated in STaRS to help teachers prepare astronomy activities from the web. Teachers will have a varying range of experience with the web so basic information is necessary:

(a) Internet access is not always available (e.g. the host site might be down or the internet provider might be offline). We therefore advise teachers to download information prior to class time.

(b) Resources may require specialist software for viewing or printing. For example, some publications in STaRS are in Adobe Acrobat PDF format, requiring the teacher to firstly download the Adobe reader.

(c) Time estimates for activities are provided by STaRS where possible to help the teacher prepare their classroom schedule.

\subsection{Local and International Resources}

Although the possibility of losing a user from a locally developed web page to a non-local page exists, STaRS was developed with the primary idea of providing the best available web education resources irrespective of the origin of the resource. With that in mind certain cautionary information had to be stated. Southern hemisphere users, when linking to sites developed in the northern hemisphere could be shown representations (images, diagrams) of objects that would be inverted to the way they are seen in the south. To illustrate this difference, STaRS provides an example of the way the well known Orion constellation is viewed from both the southern and northern hemispheres. An upside-down sun icon is used in STaRS to identify northern hemisphere sites (and highlight the southern-centric origin of STaRS!).

\subsection{Utilising the Web}

Information presented on the web should take advantage of its dynamic nature and its ability to provide quality imagery. Activities in STaRS are updated or are relevant to current astronomical events (e.g. comets, meteor showers, eclipses), present or planned telescopes, planetary and other solar system missions. High resolution colour images (e.g. from the Hubble Space Telecope, Anglo-Australian Telescope, Apollo missions, Voyager) are used to illustrate web pages and can sometimes be a more powerful educational tool than the equivalent amount of text. Images are also provided to illustrate the
STaRS activities developed by Carter Observatory. STaRS also connects many of its locally developed resource pages (including astronomical questions and answers at the 8-12 year old level) via hyperlinks, back to its activities, to promote the activities and integrate the web site.

\subsection{An Activity Example: Solar System Quiz}

A locally designed activity, Solar System Quiz \{http:// www.vuw.ac.nz/ mackie/SciTechFund/STaR S/ Educ8_12/solsys_quiz.html), has been linked with a well known planetary web site, The Nine Planets (www.anu.edu.au/Physics/nineplanets/nineplanets.html). Solar System Quiz presents an activity based on image identification of Solar System objects. Sub-images (of larger complete images) of twelve solar system objects are shown along with a list of their names to choose from. The investigators are asked to assign an object name with each sub-image. Once this is completed, a second web page is accessed showing the answers and the whole images. Students are encouraged to perform this activity before and after visiting The Nine Planets.

\section{STaRS Topical Astronomy and Space Science Studies}

The second section of STaRS, What's New in Astronomy, Astrophysics and Space Sciences? (www.vuw.ac.nz/ $\sim$ mackie/SciTechFund/STaRS/News/gen_public.html), provides hyperlinks to New Zealand and international astronomy and space science information. Current information on topical studies (e.g. dark matter, planetary searches, SETI, gamma-ray bursters, origin of the Universe, ozone depletion, aurora, Solar System missions, International Space Station, future space craft) is given at a variety of technical levels.

Importantly this part of STaRS also provides a focal point for the promotion of New Zealand astronomy and space science research via links to local research descriptions and associated international projects that New Zealand researchers are involved with, e.g. microlensing observations in astrophysics (MOA), probing lensing anomalies network (PLANET), and the Whole Earth Telescope (WET). Static links (i.e. research descriptions) are combined with dynamic or regularly updated links (i.e. IAU circulars, CNN Space News, and new HST images).

\section{Promotion, Site Feedback and Monitoring}

\subsection{Promotion: Spreading the URL}

To be effective STaRS has to be known about in schools. Therefore we have promoted STaRS through a variety of media. These have included various New Zealand education web sites, the international AstroWeb consortium (e.g. msowww.anu.edu.au/ anton/astroweb/), the Royal Society of New Zealand (www.rsnz.govt.nz/), 
email news, the New Zealand Association of Science Educators newsletter (www.nzase.org.nz/), the New Zealand astronomical society newsletters and teacher mailouts from Carter Observatory.

\subsection{STaRS Survey Form}

A web survey form (www.vuw.ac.nz/ mackie/ SciTechFund/STaRS/Educ8_12/Survey/formtest.html) is included for New Zealand teachers to complete and return. The survey questions include name; school; grade or year level; school district; how STaRS was heard of; ratings for ease of use, activities, resources; and a comments box. Over the three-month period of STaRS version 1, no completed survey forms were received. This result was disappointing since the estimated completion time of the survey was only $2-3$ minutes.

A targeted survey was sent to ten local and international astronomy teachers and educators. Seven responses were received and these showed that STaRS had achieved a 'very good' rating in both site design and content.

\subsection{Site Hits and User Statistics}

A site meter was used to monitor the usage of STaRS. As of 31 June 1999 (15 August 1999), STaRS had 755 (993) hits. New Zealand comprised 80 per cent of the users, with users from Australia, UK and USA at the 5 per cent level or less.

\section{Conclusions and Future Plans}

STaRS has proven to be a successful addition to science and technology promotion in New Zealand. It was developed on a part-time basis by a collaborative effort between researchers and educators. Further enhancements of STaRS could include web design improvements (e.g. animations and audio), ratings of all activities and resources, as well as expanding the activities presented to include those aimed at secondary (13-17 year old) school students. Whilst funding has ceased for STaRS the web site will be maintained and updated by the author on an irregular basis.

\section{Acknowledgments}

It is a pleasure to thank Tony Fisher, Frank Andrews and Mike Reid of Carter Observatory and Professor F. STaRS (www.vuw.ac.nz/ mackie/SciTechFund/STaRS/ Educ8_12/prof_stars.html) for their assistance, advice and enthusiasm in bringing STaRS to fruition. I would also like to thank the teachers, students and general public who provided critical comments on STaRS v1.0. STaRS was funded by the Science and Technology Promotion Fund of the Royal Society of New Zealand.

\section{References}

Cottrell, P. L., \& Mackie, G. 1998, Southern Stars, 38(2), 51

Leather, K., Andrews, F., Hall, R., \& Orchiston, W. 1998, in New Trends in Astronomy Teaching, ed. L. Gouguenheim et al. (Cambridge Univ. Press), p. 267

Mackie, G. 1999, Southern Stars, 38(3), 73

Pasachoff, J. M. 1998, in New Trends in Astronomy Teaching, ed. L. Gouguenheim et al. (Cambridge Univ. Press), p. 68 